\title{
Lernförderliche Arbeitsorganisation in der Industrie 4.0
}

\author{
T. Mühlbradt, P. Kuhlang und T. Finsterbusch
}

\subsection{Herausforderung "Lernen“ in der Industrie 4.0}

Cyber-Physical Production Systems (CPPS) bilden einen Kernbestandteil der angestrebten Industrie 4.0 (Kagermann et al. 2013, S. 89). Dazu ist, den Autoren zufolge, eine sozio-technische Gestaltungsperspektive erforderlich, in der Arbeitsorganisation, Weiterbildungsaktivitäten sowie Technik- und Software-Architekturen in enger wechselseitiger Abstimmung entwickelt werden (ebd., S. 57). CPPS gehen einher mit einer Informatisierung der Arbeit (Botthof 2014, S. 4), neuen Automatisierungsoptionen geistiger Arbeit (Brynjolfsson und McAfee 2011) und neuen Lernoptionen (Schuh et al. u. a., 2015, S. 84).

Aus den CPPS erwachsen neue Aufgaben- und Anforderungsprofile für Beschäftigte. Schlund et al. (2015, S. 6) kommen in einer Unternehmensbefragung zu folgendem Ergebnis: „Die Bedeutung von Qualifizierung und Kompetenzentwicklung für die Einführung von Industrie 4.0 wird als durchgängig sehr hoch eingeschätzt. Insbesondere die Bereitschaft zum lebenslangen Lernen [...] stärkeres interdisziplinäres Denken und Handeln [...] und höhere IT-Kompetenz [...] werden als notwendig angesehen." Differenzierte Szenarien sehen die Entwicklung von Tätigkeiten und Qualifikationen als offen an. So

T. Mühlbradt $(\bowtie)$

Deutsche MTM-Vereinigung e. V., Campus-Boulevard 55, 52074 Aachen, Deutschland e-mail: Thomas.Muehlbradt@dmtm.com

P. Kuhlang

Deutsche MTM-Vereinigung e. V., Elbchaussee 352, 22609 Hamburg, Deutschland

e-mail: peter.kuhlang@dmtm.com

T. Finsterbusch

Deutsche MTM-Vereinigung e. V., Eichenallee 11, 15738 Zeuthen, Deutschland e-mail: thomas.finsterbusch@dmtm.com 
Hirsch-Kreinsen (2015, S. 25): „Die vorliegenden Befunde stützen sowohl die Polarisierungsthese wie auch die Annahme einer generellen Aufwertung von Qualifikationen.“

Vor diesem Hintergrund kommt dem Wissens- und Kompetenzerwerb eine herausragende Bedeutung zu: „Die Aufgaben- und Kompetenzprofile der Mitarbeiter werden sich in Industrie 4.0 stark verändern. Das macht adäquate Qualifizierungsstrategien und eine lernförderliche Arbeitsorganisation notwendig, die lebensbegleitendes Lernen und eine arbeitsplatznahe Weiterbildung ermöglichen." (Kagermann et al. 2013, S. 6f). Schlund et al. (2015, S. 25) folgern, dass dem Thema Qualifizierung voraussichtlich ,die Rolle eines kritischen Pfades bei der Umsetzung von Industrie 4.0“ zukommt. Hirsch-Kreinsen verweist im Zusammenhang mit den angesprochenen Szenarien auf die zentrale Bedeutung des betriebsspezifischen Einführungsprozesses neuer Produktionssysteme in den Anwenderunternehmen, da sich erst dann die arbeitsorganisatorische und personelle Systemauslegung konkretisiert (ebd., 2014, S. 30). Im Folgenden werden die Konzepte Arbeitsorganisation, Lernen in der Arbeit und Lernförderlichkeit näher betrachtet. Darauf aufbauend werden Gestaltungsansätze und -strategien mit dem Schwerpunkt lernförderliche Arbeitsorganisation beschrieben.

\subsection{Lernförderlichkeit}

Das Begriffspaar Lernen im Prozess der Arbeit (LiPA) und Lernförderlichkeit ist im Zusammenhang zu betrachten. ,Mit Lernen im Prozess der Arbeit werden [...] jene Lernformen und -prozesse beschrieben, die entweder unmittelbar im Arbeitsprozess stattfinden oder sich unmittelbar auf diesen beziehen bzw. sich ihm anlagern. Ausgeklammert werden damit all jene Formen der Wissens-, Qualifikations- und Kompetenzentwicklung, die außerhalb der Erwerbsarbeit stattfinden“ (Reuther und Weiß 2003, S. 113). Arbeiten und Lernen sind in dieser Sicht keine Gegensätze: „Personalentwicklung findet vor allem in der Arbeitstätigkeit und durch Gestaltung von Arbeitstätigkeit statt“ (Ulich 2006, S. 139).

Lernen in der Arbeit kann weiter differenziert werden in ein Lernen für und aus der Arbeit (Mühlbradt u. a., 2015a, S. 3). Lernen für die Arbeit bezeichnet dann den Erwerb von Wissen und Kompetenzen für eine Aufgabe. Lernen aus der Arbeit meint aus der (längerfristigen) Durchführung der Tätigkeit entstehende, neue Wissensinhalte und Kompetenzen. Diese können dem Individuum, aber auch der Organisation zugutekommen.

Lernförderlichkeit kann demgegenüber als Fundament des Lernens in der Arbeit bezeichnet werden: „Lernförderlichkeit beschreibt die Bedingungen von Tätigkeiten, die im Arbeitsalltag bei der Arbeitsausführung Lernprozesse begünstigen bzw. lerngünstige Voraussetzungen schaffen“ (Bigalk 2006, S. 38). Ein integratives Modell, in dem LiPA und Lernförderlichkeit gemeinsam betrachtet werden, legt Mühlbradt (2014, S. 23ff) vor. Als notwendig für LiPA werden darin drei Faktoren angenommen:

- arbeitsorientiere Lernformen

- Lerngehalt der Arbeit

- lernförderliche Rahmenbedingungen 
Arbeitsorientierte Lernformen werden im Weiteren nur am Rande betrachtet. Auf den Aspekt Lerngehalt wird noch näher einzugehen sein. Als lernförderliche Rahmenbedingungen identifiziert das Modell (a. a. O., S. 24) folgende Aspekte:

- klare und verbundene Ziele auf allen Ebenen sowie Personalentwicklung als Ziel

- für LiPA geeignetes Führungsverhalten und geeignete Führungsspannen

- Verfügbarkeit von Informationen sowie geeignete Kommunikationsprozesse und -kultur

- Kapazitäten und Kompetenzen für Personaleinsatz und Personalentwicklung

- technische Infrastruktur (Hard- und Software) sowie Kompetenzen für Informationsund Kommunikations-Lösungen und Medien

- Lernbereitschaft und Bereitschaft zur Übernahme von Verantwortung

\subsection{Arbeitsorganisation}

Der Verwendung des Begriffes liegt zumeist das Arbeitssystem als konzeptueller Rahmen zugrunde. Für die (industrielle) Arbeitsgestaltung ist der Arbeitssystembegriff nach REFA (REFA 2002, S. 64-68) üblich. Danach wird ein Arbeitsbereich (ein Arbeitsplatz, eine Produktionslinie, eine Abteilung) als Arbeitssystem durch eine Systemgrenze von seiner Umwelt abgegrenzt. Das Arbeitssystem beinhaltet die Elemente: Arbeitsaufgabe, Arbeitsablauf, Eingabe, Ausgabe, Mensch, Betriebs- und Arbeitsmittel sowie Umgebungseinflüsse. Zwischen diesen Elementen gibt es vielfältige Beziehungen.

Für jedes Arbeitssystem besteht eine Arbeitsorganisation. Diese definiert Heeg (1991, S. 17) wie folgt: ,Arbeitsorganisation ist das Schaffen eines aufgabengerechten, optimalen Zusammenwirkens von arbeitenden Menschen, Betriebsmitteln, Informations- und Arbeitsgegenständen durch zweckgerichtete Gliederung der Arbeitsaufgabe, der Aufgabenteilung zwischen den Menschen und Betriebsmitteln, der Gestaltung von Information, Kommunikation und Arbeitszeit.“ Knapper formuliert Hirsch-Kreinsen (2014, S. 13): „die arbeitsteilige Strukturierung von Aufgaben und Tätigkeiten in horizontaler und hierarchischer Hinsicht und damit verbundene Gestaltung von Kooperation und Kommunikation zwischen den im und am System Beschäftigten.“

Erweitert man im Hinblick auf die technologischen Möglichkeiten der Industrie 4.0 diese Definitionen noch um Beziehungen zwischen Betriebsmittel, Informationsmittel und Arbeitsgegenstand, so kann die Arbeitsorganisation wie in der Abb. 14.1 gezeigt dargestellt werden.

Im Kern legt die Arbeitsorganisation fest, wie die Gesamtaufgabe des Arbeitssystems zwischen Menschen beziehungsweise Mensch und Maschine aufgeteilt wird und überbrückt anschließend die daraus entstehenden Schnittstellen durch Kooperations- und Kommunikationsstrukturen und -abläufe. 


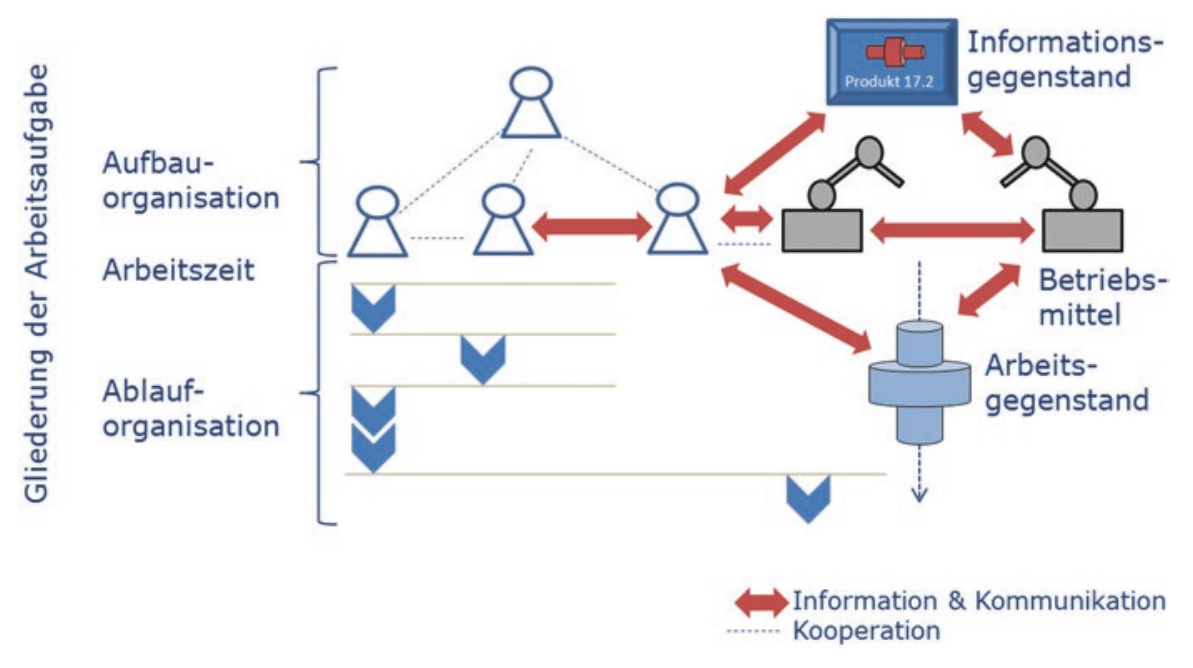

Abb. 14.1 Arbeitsorganisation (Mühlbradt 2015, S. 228)

Die Arbeitsorganisation ist im konkreten Fall in aller Regel nicht determiniert durch die eingesetzte Technologie, sodass auch an vergleichbaren Arbeitsplätzen unterschiedliche Bedingungen existieren können (z. B. Bigalk 2006, S. 151). Arbeitsorganisatorische Lösungen spiegeln in starkem Maße betrieblich tradierte Paradigmen der Arbeitsorganisation sowie Ziele, Prinzipien und Erfahrungen wider. ${ }^{1}$ Auf diesen Aspekt wird später mit Blick auf „Lernkulturen“ noch eingegangen.

\subsection{Potenziale der Arbeitsorganisation in Bezug auf Lernförderlichkeit}

Für den vorliegenden Gegenstand lassen sich vor allem folgende Aspekte herausarbeiten:

- Die Arbeitsorganisation definiert Tätigkeiten und damit Arbeitsinhalte sowie Grenzen zwischen Tätigkeiten. Eine „Tätigkeit“ stellt dann die Summe der einer bestimmten Person dauerhaft übertragenen Arbeitsaufgaben dar. ${ }^{2}$ Neben der horizontalen Dimension (Anzahl verschiedener Aufgaben) bestimmt die vertikale Dimension, ob neben der Aufgabenausführung jeweils auch dispositive Aufgabenanteile existieren, also

\footnotetext{
${ }^{1}$ Die grundsätzliche Gestaltungsfreiheit der Arbeitsorganisation tritt auch in Rechtsnormen auf, so in der Gewerbeordnung $§ 106$,Weisungsrecht des Arbeitgebers“: ,Der Arbeitgeber kann Inhalt, Ort und Zeit der Arbeitsleistung nach billigem Ermessen näher bestimmen [...]“

${ }^{2}$ In einem weiteren Sinne kann „Tätigkeit“ auch verstanden werden als die Summe der Eindrücke und Erfahrungen, die jemand im Arbeitsleben hat. Dies schließt dann nicht arbeitsbezogene Kommunikation, das Erleben organisationaler Veränderungen etc. mit ein.
} 
Zielsetzung, Planung und Kontrolle dazugehören. Diese Arbeitsteilung findet zwischen Menschen im Arbeitssystem, aber auch zwischen Menschen und Maschinen statt, wobei neben manueller Arbeit auch zunehmend geistige Arbeit automatisierungsfähig wird. Der Zusammenhang zur Lernförderlichkeit ist offensichtlich: Aufgaben, die nicht mir, sondern jemand anderem - oder einer Maschine - übertragen wurden, stellen nur geringe Lernanforderungen und -chancen für mich dar.

- Die Arbeitsorganisation legt Umfang und Form der zur Aufgabenerfüllung notwendigen Kommunikation zwischen Menschen im System und am System (d. h. zwischen System und Umwelt) fest. Zwischen den Polen ,,isolierte Einzelarbeit“ und ,selbstgesteuerte Gruppenarbeit" bestimmt sie damit analoge und digitale Vernetzungsanforderungen zwischen Menschen in der Arbeitstätigkeit. In dem Maße, wie eine Prozessorientierung vorliegt, also das Arbeitssystem als integrativer Bestandteil einer übergreifenden Wertschöpfungskette angelegt ist, ergeben sich weitere wichtige Kommunikationsbeziehungen zur Umwelt in Form von vor- und nachgelagerten Arbeitssystemen, Kunden und Lieferanten. Prozesstransparenz und Rückmeldungen werden dadurch wesentlich (Hammer und Champy 1993).

- Die Arbeitsorganisation beeinflusst mit den zuvor genannten Bereichen wesentlich den Lerngehalt der Arbeitstätigkeit. Frieling u. a. (2006, S. 57) identifizieren als tätigkeitsbezogene Schlüsselmerkmale der Lernförderlichkeit Komplexität und Selbständigkeit. Komplexität entsteht aus Anforderungsvielfalt und -tiefe und den damit einhergehenden Wissens- und Kompetenzanforderungen. Selbständigkeit (Autonomie) entsteht aus Handlungs- und Entscheidungsspielräumen und der damit verbundenen Verantwortung. Bergmann und Richter (2003, S. 41) betonen die motivationale Seite und sehen „Aufgabenbedeutung sowie Rückmeldungen und die Transparenz von Informationsflüssen“ als bedeutsam für die „Entwicklung intrinsischer arbeitsorientierter Lernmotivation“.

- Neben den objektiven Gegebenheiten sind individuelle Unterschiede zwischen Personen bedeutsam. Persönliche Werte, Bedürfnisse und Fähigkeiten interagieren mit objektiven Tätigkeitsmerkmalen (z. B. Oldham und Hackmann 2010, S. 467). Die erlebte Komplexität einer Aufgabe ist eben auch abhängig von Wissen und Fähigkeiten des Durchführenden; die subjektive Aufgabenbedeutung ist abhängig von persönlicher Wahrnehmung und normativer Bewertung objektiver Gegebenheiten. Damit ist weder eine bestimmte Lösung für alle Menschen (zu einem Zeitpunkt) gleichermaßen geeignet, noch stellt eine Verstärkung bestimmter Merkmale (,,mehr ist besser“) einen Wert an sich dar.

\subsection{Gestaltungsansätze lernförderlicher Arbeitsorganisation für Industrie 4.0}

Auf dieser Grundlage können nun Hinweise für Gestaltungsansätze gegeben werden. Diese Hinweise stellen keine vollständige Auflistung dar und können jeweils nur angerissen werden. Für eine vertiefte Beschäftigung muss auf weiterführende Literatur verwiesen werden. 


\section{Schnittstellen arbeitsorientierter Lernformen zur Arbeitsorganisation}

Eine systematische Aufarbeitung von arbeitsnahen Lernformen findet sich in Mühlbradt, u. a. (2015a). Bedeutsame Schnittstellen zur Arbeitsorganisation besitzen vor allem die Qualifizierende Arbeitsgestaltung sowie die Integration von Arbeits- und Lernaufgaben.

Die Qualifizierende Arbeitsgestaltung beteiligt Beschäftigte im und am Arbeitssystem an der Planung und Gestaltung des Arbeitssystems. Für vorwiegend manuelle Arbeitstätigkeiten haben sich Methodenräume bewährt. In ihnen werden Strukturen und Abläufe, Arbeitsmittel und Informationsmittel gemeinsam mit Fachexperten unter Zuhilfenahme geeigneter Verfahren (z. B. Card Board Engineering) entworfen und gestaltet. Solche Methodenräume nutzen nicht nur das Fach- und Erfahrungswissen der Beschäftigten, sondern erweitern auch deren Kenntnisse und Fähigkeiten - sie haben eine qualifizierende Wirkung. Für vorwiegend geistige Arbeit mit Mensch-Maschine-Interaktion stellt das Rapid Collaborative Prototyping einen Ansatz dar. Hier werden schnelle, iterative Gestaltungsschritte von Software unter Einbezug der späteren Anwender unternommen - ebenfalls mit qualifizierender Wirkung.

Eine Integration von Arbeits- und Lernaufgaben sehen verschiedene arbeitsintegrierte Lernformen vor. Arbeitsaufgaben werden als Lernaufgaben organisiert und bestimmten Personen in einem Lernprozess zugewiesen; die Lernaufgabengestaltung orientiert sich an den vorliegenden oder geplanten Arbeitsaufgaben. Damit kommt Verfahren der objektiven und subjektiven Arbeitsanalyse eine zentrale Funktion zu. Objektive Analysen ermitteln beispielsweise Aufgabeninhalte und Anforderungen aus Expertensicht. Subjektive Analysen ergänzen erlebte Arbeitsmerkmale und subjektive mentale Modelle von Abläufen oder technischen Systemen und sind besonders geeignet, Lernvoraussetzungen aufzuzeigen.

\section{Lerngehalt von Tätigkeiten Arbeitsstrukturierung}

bezeichnet die dauerhafte Veränderung von Tätigkeiten durch horizontale (job enlargement) oder vertikale (job enrichment) Erweiterung von Tätigkeiten. Hinzu kommt der systematische Wechsel zwischen Aufgaben (job rotation). Ähnliche Effekte zeigen multifunktionale Teamstrukturen. Dieser Ansatz baut auf Rollenkonzepten und einer Matrixorganisation auf und sieht wechselnde Teamarbeitsformen in festen Arbeitsgruppen, Geschäftsprozessen oder Projekten als alternierende oder parallele Organisation vor.

Die Idee der differentiell-dynamischen Arbeitsorganisation (Ulich 1978) besteht darin, bestimmte arbeitsorganisatorische Setzungen einerseits zwischen Personen zu differenzieren (interindividuell) und andererseits Veränderungen über die Zeit innerhalb einer Person (intraindividuell) zuzulassen. Gerade CPPS schaffen hierfür interessante technische Möglichkeiten. So ist beispielweise vorstellbar, Aufgaben mit Anwendungssoftware in Form sogenannter „Apps“ zu koppeln und diese jeweils differenziert für individuelle mobile Endgeräte freizuschalten. Einen ähnlichen, aber noch erweiterten Ansatz stellt das Job Crafting dar (Tims und Bakker 2010). Hier werden Anforderungen (job demands) je nach Bedarf herauf- oder herabgesetzt und Ressourcen (job resources) nach Bedarf erhöht. Dies kann relativ dauerhaft oder auch nur kurzfristig geschehen. Das Job Crafting liegt 
im Ermessensspielraum der betroffenen Person und wird durch sie selbst vorgenommen. ${ }^{3}$ Auch hier bieten CPPS neue Optionen, in dem Aufgaben an eine Maschine delegiert oder zurückgenommen werden können oder soziale Unterstützung durch Kollegen über digitale Medien angefordert werden beziehungsweise erfolgen kann.

Der Lerngehalt wird weiterhin über die systematische Beteiligung an Standardisierungs- und Problemlösungsprozessen beeinflusst, die neben den Kernaufgaben bestehen (z. B. Röben 2006; Springer und Meyer 2006; Liker und Meier 2006; Rother 2009). Neben einem Job Enrichment ergeben sich erweiterte Kommunikationsanforderungen sowie die motivational bedeutsame Erfahrung der Selbstwirksamkeit (Deci und Ryan 1985; Frieling u. a., 2006, S. 92), auch wenn der Lerngehalt der Kernarbeit selbst nicht verändert werden kann oder soll.

\section{Kooperation und Kommunikation im und am Arbeitssystem}

Das Konzept der Regelkommunikation implementiert geplante und regelmäßige Kommunikationssituationen als definierten Rahmen für den Informationsaustausch im Arbeitssystem selbst, beziehungsweise zwischen dem Arbeitssystem und seiner Umwelt. Dieser Rahmen ist zeitlich, personell und methodisch vorbestimmt und schafft damit eine Balance zwischen häufiger, zeitnaher Kommunikation und Aufwand (z. B. Schuh et al. u. a., 2014, S. 288). Communities of Practice (Wenger 1998) sind Gruppen von Personen, die ein gemeinsames arbeitsbezogenes Interesse aufweisen und Wissen und Kompetenzen durch häufige Interaktion erweitern. Diese Gruppen sind Arbeitssystem-übergreifend zusammengesetzt. Hier bieten internetbasierte soziale Medien innovative Gestaltungsmöglichkeiten (z. B. Bohlander et al. u. a., 2011). Schließlich lassen sich in CPPS dafür auch Mensch-Maschine-Kombinationen denken. So sollen sich Produktionssysteme als ,Social Machines" automatisch über Cloud-basierte Dienste verbinden und sich situationsabhängig die benötigten menschlichen Experten suchen (Kagermann et al. 2013, S. 69).

\subsection{Lernförderlichkeit als Strategie}

In den bisherigen Ausführungen wurde die Arbeitsorganisation als zu gestaltende Lösung betrachtet. An dieser Stelle soll über diese Sichtweise hinausgegangen werden.

In der betrieblichen Praxis ist eine zunehmende Variabilität arbeitsorganisatorischer Lösungen festzustellen. So bestehen bedeutsame Unterschiede selbst zwischen verschiedenen Produktionslinien desselben Produkts. Innerhalb von Arbeitssystemen folgen Veränderungen immer schneller aufeinander, sodass mittel- bis längerfristig konstante Arbeitsorganisationen seltener angetroffen werden. Schließlich ist es aufgrund der Heterogenität und Komplexität der Arbeitsorganisation immer schwieriger zu bestimmen, ob gleichlautende Lösungen immer auch faktisch identisch sind.

\footnotetext{
${ }^{3}$ Es sei auf den daraus entstehenden Abstimmungsaufwand zwischen betrieblichen und persönlichen Zielen hingewiesen.
} 
Darüber hinaus ist von einer gewissen Pfadabhängigkeit arbeitsorganisatorischer Veränderungsprozesse auszugehen. Lorenz und Valeyre (2005) kommen auf Basis einer inferenzstatistischen Auswertung von EWCS-Daten ${ }^{4} \mathrm{zu}$ dem Ergebnis, dass sich vier grundlegende Paradigmen der Arbeitsorganisation finden lassen, die sich hinsichtlich des Stellenwertes sowie der Art und des Ausmaßes von Lernen deutlich unterscheiden (vgl. Unger 2012; Mühlbradt u. a., 2015b). Da solche Paradigmen langfristig bestehen, ist davon auszugehen, dass sie verschiedene Lernkulturen etablieren. ${ }^{5}$ Daran wird deutlich, dass identische arbeitsorganisatorische Lösungen auch aus organisationaler Perspektive nicht in allen Situationen gleichwertig sein können (vgl. Pfäfflin und Rehberg 2008, S. 97).

Vor dem Hintergrund von Variabilität und Pfadabhängigkeit ist es hilfreich, die Arbeitsorganisation in Anlehnung an Weick (1985) auch als „Prozess des Organisierens“ zu verstehen. Dieser Prozess ist selbst ein Lernprozess, in dem verschiedene Lösungen innerhalb eines Paradigmas erprobt und verändert werden oder ein längerfristiger Wandel von einem alten zu einem neuen Paradigma stattfindet. Aus dieser Perspektive kann eine lernförderliche Arbeitsorganisation als umfassendes betriebliches Lernprojekt angelegt werden, bei dem gute und schlechte Erfahrungen mit bestimmten Lösungen systematisch erfasst und verarbeitet werden und sich eine individuelle betriebliche Praxis herausbildet. Notwendig ist dazu ein leitendes Instrumentarium, welches von konkreten arbeitsorganisatorischen Lösungen abstrahiert und ein evidenzbasiertes Voranschreiten unterstützt. Dieser Aspekt soll abschließend näher betrachtet werden.

Mit der „Wissensbilanz made in Germany“ (Alwert et al. 2008) liegt ein Instrument vor, um das Intellektuelle Kapital einer Organisation zu erfassen und zu bewerten. Unterschieden werden dabei die Kapitalarten Struktur-, Human- und Beziehungskapital, die betriebsindividuell auszufüllen und zu bewerten sind. Dieser Ansatz kann für den „Prozess des Organisierens von Lernförderlichkeit“ fruchtbar gemacht werden, indem systematisch und periodisch Veränderungen beim Intellektuellen Kapital erhoben und bewertet werden. Zur Ausformung der drei Kapitalarten im Hinblick auf Lernförderlichkeit kann der nachstehende Vorschlag eine Orientierung geben:

Strukturkapital

- Integration von Arbeitsgestaltung und Personalentwicklung

- Quantität und Qualität arbeitsorientierter Lernlösungen

- Individualisierung von Tätigkeitsinhalten

- Durchlässigkeit von Tätigkeitsgrenzen

- Verbreitung und Qualität der Regelkommunikation

\footnotetext{
${ }^{4}$ European Working Conditions Survey.

${ }^{5}$ Dabei kann Lernkultur mit Schmidt-Rathjens (2007, S. 4) verstanden werden als „,... Gesamtheit der Wertvorstellungen, Denkmuster, Handlungsweisen und Rahmenbedingungen einer Organisation und ihrer Mitglieder hinsichtlich der Förderung von Lernen und Entwicklung von Kompetenzen.“
} 
Humankapital

- lernförderliches Führungsverhalten

- Selbständigkeit der Beschäftigten

Beziehungskapital

- Transparenz

- wahrgenommene Qualität und Bedeutung (Sinnhaftigkeit) der Arbeit

CPPS als sozio-technische Systeme ermöglichen und erfordern lebensbegleitendes Lernen der Beschäftigten. Die Informatisierung der Arbeit und die Verfügbarkeit multimedialer Mensch-Computer-Schnittstellen schaffen neue Gestaltungsoptionen für das individuelle und organisationale Lernen. Lernförderliche industrielle Arbeitssysteme benötigen aber darüber hinaus geeignete arbeitsorganisatorische Konzepte und Organisationsstrategien als Fundament des Lernens in der Arbeit.

\section{Literatur}

Alwert, K., Bornemann, M., \& Will, M. (2008). Wissensbilanz - Made in Germany. Bundesministerium für Wirtschaft und Energie, Berlin.

Bergmann, B., \& Richter, F. (2003). Berufliche Kompetenzentwicklung in der veränderten Arbeitswelt. Wirtschaftspsychologie 2003, Ausgabe 1, S.1996-1998.

Bigalk, D. (2006). Lernförderlichkeit von Arbeitsplätzen - Spiegelbild der Organisation? Kassel.

Bohlander, H., Gebauer, T., Mühlbradt, T., \& Kivikas, M. (2011). Ressource Wissen nutzen - Wissensmanagement strategieorientiert entwickeln. Schriftenreihe des Forschungsinstituts Betriebliche Bildung (f-bb) gemeinnützige $\mathrm{GmbH}$ (Bd. 48). Bielefeld.

Botthof, A. (2014). Einordnung und Hintergründe. In Bundesministerium für Wirtschaft und Energie (Hrsg.), Zukunft der Arbeit in Industrie 4.0 (S. 4-6). Berlin: Springer.

Brynjolfsson, E., \& McAfee, A. (2011). Race against the machine: How the digital revolution is accelerating innovation, driving productivity, and irreversibly transforming employment and the economy. Lexington, MA.: Digital Frontier Press.

Deci, E. L., \& Ryan, R. M. (1985). Intrinsic motivation and self-determination in human behavior. New York: Plenum.

Frieling, E. u. a. (2006). Lernen durch Arbeit. Entwicklung eines Verfahrens zur Bestimmung der Lernmöglichkeiten am Arbeitsplatz. Münster: Waxmann Verlag GmbH.

Hammer, M., \& Champy, J. (1993). Reengineering the corporation: A manifesto for business Revolution. New York: Harper Business.

Heeg, F.-J. (1991). Moderne Arbeitsorganisation. 2. Aufl. Darmstadt: Hanser.

Hirsch-Kreinsen, H. (2014). Wandel von Produktionsarbeit - „Industrie 4.0“. Soziologisches Arbeitspapier Nr. 38/2014. Dortmund.

Hirsch-Kreinsen, H. (2015). Digitalisierung von Arbeit: Folgen, Grenzen und Perspektiven. Soziologisches Arbeitspapier Nr. 43/2015. Dortmund.

Kagermann, H., Wahlster, W., \& Helbig, J. (2013). Umsetzungsempfehlungen für das Zukunftsprojekt Industrie 4.0. Promotorengruppe Kommunikation der Forschungsunion Wirtschaft Wissenschaft, Frankfurt a. M. 
Liker, J. K., \& Meier, D. P. (2006). The Toyota way fieldbook. New York: McGraw-Hill.

Lorenz, E., \& Valeyre, A. (2005). Organisational innovation, human resource management and labour market structure: A comparison of the EU-15. Journal of Industrial Relations, 47(4), 424-442.

Mühlbradt, T. (2014). Was macht Arbeit lernförderlich? - Eine Bestandsaufnahme. In Deutsche MTM-Vereinigung e. V. (Hrsg.), MTM-Schriften Industrial Engineering, Ausgabe 1. Berlin: Deutsche MTM-Vereinigung e. V.

Mühlbradt, T. (2015). „Engineering lernförderlicher Industrieller Arbeitssysteme“. In P. Kuhlang (Hrsg.), Modellierung menschlicher Arbeit im Industrial Engineering (S. 209-244). Stuttgart: Ergonomia Verlag.

Mühlbradt, T., Senderek, R., Rodenhauser, T., \& Saupp, L. (2015a). Arbeitsorientierte Lernlösungen für Industrielle Arbeitssysteme: Lernen für die Arbeit. In Deutsche MTM-Vereinigung e. V. (Hrsg.), MTM-Schriften Industrial Engineering, Ausgabe 2. Berlin: Deutsche MTM-Vereinigung e. V.

Mühlbradt, T., Kuhlang, P., \& Senderek, R. (2015b). Lernkultur als Kernkompetenz für das arbeitsnahe Lernen in der Industrie 4.0. In ABWF (Hrsg.), Lernförderliche Arbeitsgestaltung Arbeitsförderliches Lernen. ABWF-Bulletin, (im Druck).

Oldham, G. R., \& Hackman, J. R. (2010). Not what it was and not what it will be: The future of job design research. Journal of Organizational Behavior, 31(2-3), 463-479.

Pfäfflin, H., \& Rehberg, F. (2008). Erfahrungsförderliche Arbeitsgestaltung. In W. Adami u. a. (Hrsg.), Montage braucht Erfahrung (S. 96-115). München und Mehring: Rainer Hampp Verlag.

REFA. (Hrsg.). (2002). Ausgewählte Methoden zur Prozessorientierten Arbeitsorganisation. Darmstadt.

Reuther, U., \& Weiß, R. (2003). Der Programmbereich „Lernen im Prozess der Arbeit“. In Arbeitsgemeinschaft Betriebliche Weiterbildungsforschung e. V. (Hrsg.), Zwei Jahre „Lernkultur Kompetenzentwicklung“. QUEM-report Schriften zur beruflichen Weiterbildung, Heft 79 (S. 91-138). Berlin: ESM Satz und Grafik GmbH.

Röben, P. (2006). Betriebliche Weiterbildung und einfache Arbeit. In U. Clement \& M. Lacher (Hrsg.), Produktionssysteme und Kompetenzerwerb (S.157-180). Stuttgart: Franz Steiner Verlag.

Rother, M. (2009). Toyota Kata: Managing people for improvement, adaptiveness and superior results. New York: Mcgraw-Hill.

Schlund, S., Hämmerle, M., \& Strölin, T. (2015). Industrie 4.0 - Eine Revolution der Arbeitsgestaltung. Stuttgart: Ingenics AG.

Schmidt-Rathjens, C. (2007). Spezifische Bedingungen von KMU bezüglich der Entwicklung und Erfassung der betrieblichen Lernkultur. Die Lernkultur-Checkliste (LKC-KMU). QUEM-Materialien, N79, Berlin.

Schuh, G., Potente, T., Thomas, C., \& Hauptvogel, A. (2014). Steigerung der Kollaborationsproduktivität durch cyber-physische Systeme. In T. Bauernhansl, M. ten Hompel, \& B. Vogel-Heuser (Hrsg.), Industrie 4.0 in Produktion, Automatisierung und Logistik (S. 276-295). Wiesbaden: Springer.

Schuh, G., Gartzen, T., Rodenhauser, T., \& Marks, A. (2015). Promoting work-based learning through industry 4.0. In Procedia CIRP (S. 82-87). Amsterdam: Elsevier

Springer, R., \& Meyer, F. (2006). Flexible Standardisierung von Arbeitsprozessen. In U. Clement \& M. Lacher (Hrsg.), Produktionssysteme und Kompetenzerwerb (S. 43-54). Stuttgart: Franz Steiner Verlag.

Tims, M., \& Bakker, A. B. (2010). Job crafting: Towards a new model of individual job redesign. SA Journal of Industrial Psychology, 36(2), 1-9.

Ulich, E. (1978). Über mögliche Zusammenhänge zwischen Arbeitstätigkeit und Persönlichkeitsentwicklung. Psychosozial, (1), 44-63. 
Ulich, E. (2006). Lern- und Entwicklungspotentiale in der Arbeit - Beiträge der Arbeits- und Organisationspsychologie. In K. Sonntag (Hrsg.), Personalentwicklung in Organisationen (S. 138176). Göttingen: Hogrefe-Verlag.

Unger, H. (2012). Lernen im Prozess der Arbeit - zur Aktualität einer alten Idee. In R. Schimweg u. a. (Hrsg.). Partizipation und Führung (S.97-110). Marburg: Tectum Verlag.

Weick, K. (1985). Der Prozess des Organisierens. Frankfurt a M: Suhrkamp.

Wenger, E. (1998). Communities of practice: learning, meaning, and identity. Cambridge: Cambridge University Press.

Open Access Dieses Kapitel wird unter der Creative Commons Namensnennung 4.0 International Lizenz (http://creativecommons.org/licenses/by/4.0/deed.de) veröffentlicht, welche die Nutzung, Vervielfältigung, Bearbeitung, Verbreitung und Wiedergabe in jeglichem Medium und Format erlaubt, sofern Sie den/die ursprünglichen Autor(en) und die Quelle ordnungsge-mäß nennen, einen Link zur Creative Commons Lizenz beifügen und angeben, ob Änderungen vorgenommen wurden.

Die in diesem Kapitel enthaltenen Bilder und sonstiges Drittmaterial unterliegen ebenfalls der genannten Creative Commons Lizenz, sofern sich aus der Abbildungslegende nichts anderes ergibt. Sofern das betreffende Material nicht unter der genannten Creative Commons Lizenz steht und die betreffende Handlung nicht nach gesetzlichen Vorschriften erlaubt ist, ist für die oben aufgeführten Weiterverwendungen des Materials die Einwilligung des jeweiligen Recht-einhabers einzuholen.

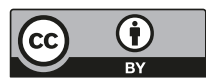

